\title{
CERN ELENA project progress report
}

\author{
Wolfgang Bartmann"1, Pavel Belochitskii ${ }^{1}$, Horst Breuker ${ }^{1}$, François Butin ${ }^{1}$, C. Carli', Tommy Eriksson ${ }^{1}$, \\ Walter Oelert' ${ }^{2}$, Stephan Maury ${ }^{1}$, Sergio Pasinelli', Gerard Tranquille ${ }^{1}$ \\ ${ }^{1}$ CERN, Switzerland \\ ${ }^{2}$ Johannes-Gutenberg-Universitaet Mainz, Germany
}

\begin{abstract}
The Extra Low Energy Antiproton ring (ELENA) is a CERN project aiming at constructing a $30 \mathrm{~m}$ circumference synchrotron to further decelerate antiprotons from the Antiproton Decelerator (AD) from $5.3 \mathrm{MeV}$ to $100 \mathrm{keV}$. The additional deceleration complemented by an electron cooler to reduce emittances will allow the existing $\mathrm{AD}$ experiments to increase substantially their antiproton capture efficiencies and render new experiments possible. The ELENA design is now well advanced and the project has entered the construction stage, in particular for what concerns the infrastructure. Installation of the machine components is foreseen during the second half of 2015 and beginning of 2016 followed by ring commissioning until the end of 2016. New electrostatic transfer lines to the experiments will be installed and commissioned during the first half of 2017 followed by the first physics operation with AD/ELENA end of 2017. Main ELENA related infrastructure progresses as well as the status of the project are reported.
\end{abstract}

\section{The ELENA Project}

The ELENA (Extra Low Energy Antiproton) project is a small size (30.4 $\mathrm{m}$ circumference) synchrotron with hexagonal shape, to be built at CERN. It will further decelerate and cool the antiprotons delivered by the existing CERN AD (Antiproton Decelerator) machine, from the energy of $5.3 \mathrm{MeV}$ down to a final energy of $100 \mathrm{keV}$.

The current AD physics programme consists in trapping antiprotons to create anti-hydrogen atoms after recombination with positrons. The ultimate physics goal is to perform spectroscopy on these anti-atoms at rest and to compare the effect of the gravitational force on matter and antimatter.

With the current set-up, most (99.9\%) of the antiprotons decelerated in the AD are lost due to the final deceleration process of the experiments using the antiprotons.

By employing a ring equipped with beam cooling, an important increase in phase-space density and high experiment injection efficiency can be obtained, resulting in an increased number of trapped antiprotons.

With the construction of the ELENA ring, the AD experiments expect efficiency improvements of up to two orders of magnitude in the number of usable antiprotons.

In addition, ELENA will be able to deliver beams almost simultaneously to up to four experiments resulting in an essential gain in total beam time for each experiment. The construction of the new facility also opens up the possibility of accommodating an extra experimental zone in the AD hall. 
The ELENA machine will use a RF (Radio Frequency) cavity to decelerate the antiprotons, and an electron cooler to keep the beam emitance low.

The ELENA project comes in the wide context of the AD facility consolidation project, which includes the consolidation of the AD machine (renewal of magnets, stochastic cooling, kickers etc.), the renewal of the $\mathrm{AD}$ hall and infrastructure (upgrade of cranes, hall cooling system, relocation of existing mechanical workshop), the relocation of the $\mathrm{AD}$ experiments control room in a new adjacent building, the installation of a new external building to provide more space for preparation, cleaning, storage of the experimental equipment. Also the new experiment BASE was just installed in 2014, that will use the $\mathrm{AD}$ beam, and that has to be added to the list of experiments to be served by the ELENA beam.

\section{ELENA layout}

The ELENA machine (see Figure 1) is constituted by:

- an hexagonal shape synchrotron

- a transfer line from the AD machine

- $\quad$ an ion source and its transfer line

- $\quad$ ejection lines towards the existing AD experiments (ALPHA, ASACISA, ATRAP, AeGIS and BASE) plus at least one new experiment (GBAR).

The design of the areas (see Figure 2) keeps the possibility to later add one "large" experiment, plus one "high energy" experiment, using directly the $\mathrm{AD}$ beam either at the injection energy (500 MeV) or with a decelerated beam $(5.3 \mathrm{MeV})$.

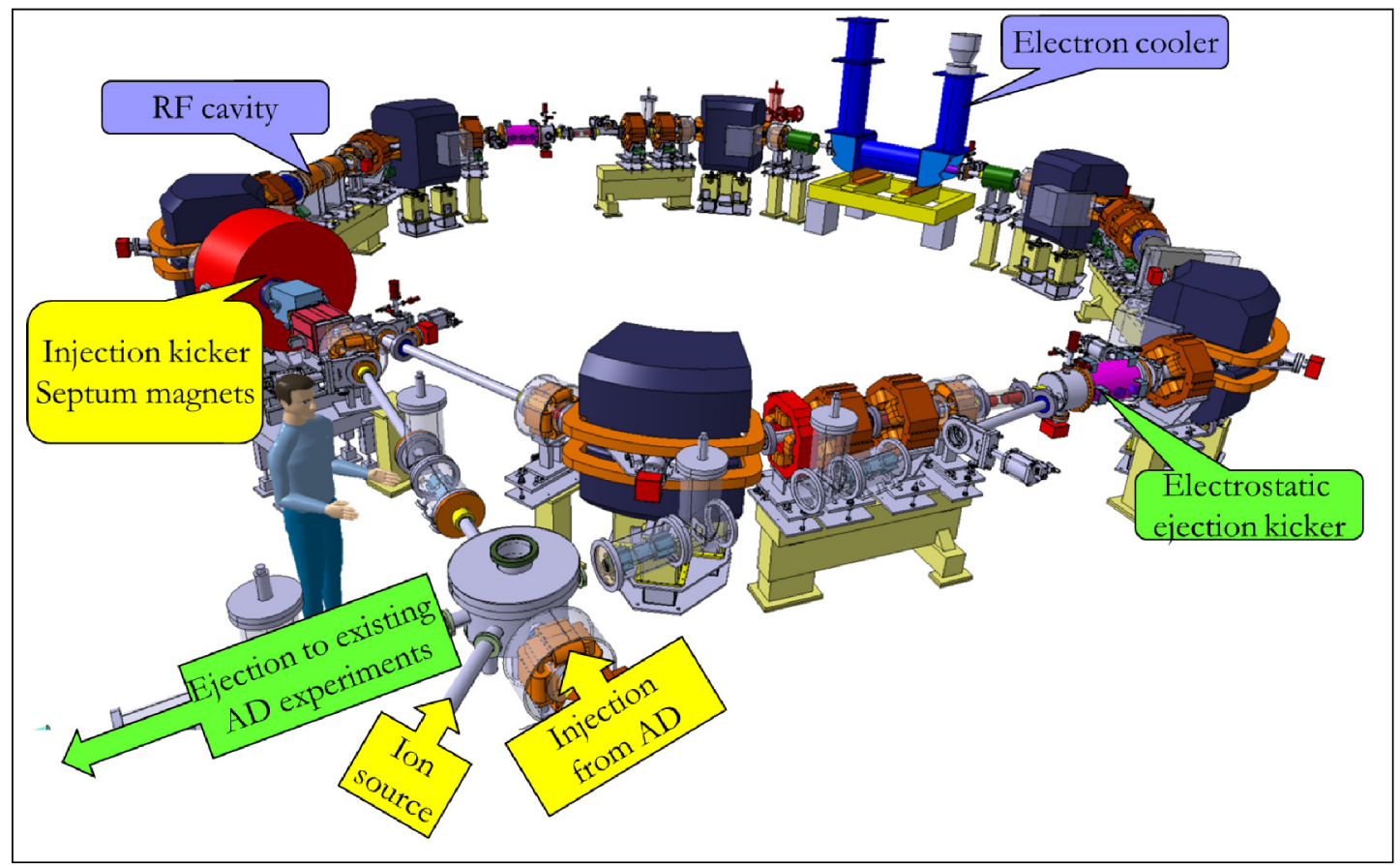

Figure 1. The ELENA machine layout. 


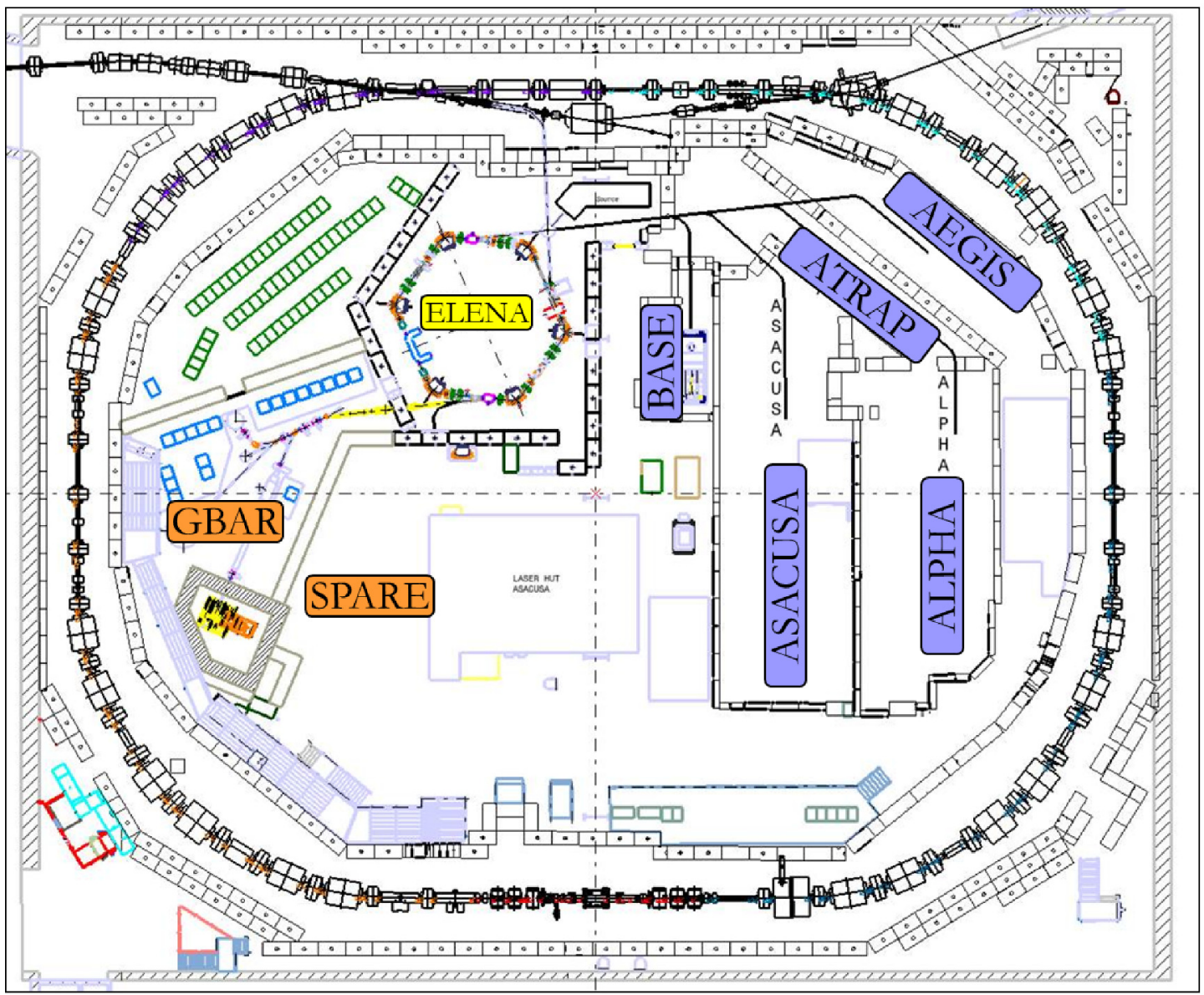

Figure 2. The ELENA machine, the existing and future experiments in the $\mathrm{AD}$ hall.

\section{ELENA project status}

Among the various challenges of the ELENA project, one of the most complex one that will impact all the elements of the machine, is the lack of space: indeed despite its small size $(30.4 \mathrm{~m}$ circumference), it nevertheless includes all the usual elements of a large scale synchrotron, plus some less usual ones, directly linked to the decelerating nature of this machine. On top of this, the ELENA machine has to be fitted inside the already rather crowded AD hall, within the span of the existing AD ring. To this aim, a fraction of the existing infrastructure in the AD hall is being relocated in newly built adjacent buildings (control room building and annex technical building). In the present chapter, we give an overview of the project main components status at the time of writing this document.

\subsection{Beam cooling using the electron cooler}

The deceleration of the AD antiprotons in the ELENA machine will necessitate cooling down the beam to keep the emittances low. The antiprotons will be decelerated in ELENA in two stages: first to a momentum of $35 \mathrm{MeV} / \mathrm{c}$ (corresponding to a kinetic energy of $650 \mathrm{keV}$ ) and then to $13.7 \mathrm{MeV} / \mathrm{c}$ (corresponding to a kinetic energy of $100 \mathrm{keV}$ ). Electron cooling will be essential at these two momenta in order to obtain the small emittance beams needed for either further deceleration or extraction to the experiments. 
An electron gun will produce a cold $\left(\mathrm{T}^{\perp}<0.1 \mathrm{eV}, \mathrm{T} / /<1 \mathrm{meV}\right)$ and relatively intense electron beam ( $\mathrm{T}^{\perp}$ is the temperature of the electron beam in the direction perpendicular to the beam axis, $\mathrm{T} / /$ is the temperature in the direction parallel to the beam axis). A crucial point of the design of the electron cooler is the quality of the longitudinal magnetic field guiding the electrons from the gun to the collector and in particular the quality of the magnetic field in the interaction region of the drift solenoid. The transverse components of the longitudinal field in the drift solenoid must be kept small $(\mathrm{B} \perp / \mathrm{B} / /<5 \times 10-4$, with $\mathrm{B} / /=100$ Gauss, $\mathrm{B} \perp$ being the magnetic field component perpendicular to the beam axis and $\mathrm{B} / /$ the field component parallel to the beam axis), to ensure a minimal perturbation to the electron beam transverse temperature.

The magnetic system of the electron cooler that is fairly complex and quite challenging given the accuracy required is now contracted to industry, while the internal vacuum system is being designed and will be produced at CERN.

\subsection{Beam Vacuum system}

The ELENA vacuum system is designed in such a way as to guarantee a sufficiently long antiproton lifetime and reduced beam blow-up caused by interaction of the antiproton beam injected from the $\mathrm{AD}$. In order to guarantee adequate conditions, calculations indicate that the average pressure should be below $3 \times 10^{-12}$ Torr.

In order to achieve this, and considering the limited amount of free space left along the machine lattice for the installation of lumped vacuum pumps, and also considering the extensive experience and expertise in the field of NEG coating available at CERN, the decision has been taken to apply the NEG-coating technology around the ring wherever possible.

As a consequence, the ELENA ring will be fully bakeable $\left(300^{\circ} \mathrm{C}\right)$, with NEG coated chambers.

Permanent bake-out equipment will be installed in the magnets. Mobile mechanical pumping groups and mobile diagnostics (RGA) will be used.

\subsection{Modification of the AD line 7000}

$\mathrm{AD}$ line 7000 is currently used to transfer the antiprotons ejected from $\mathrm{AD}$ to the existing experiments. It had to be modified to enable steering the beam towards ELENA. This included changing two quadrupoles and making the line more compact along the beam direction. This job was completed for the AD re-start in July 2014.

\subsection{ELENA magnets}

The ring magnet system of ELENA consists of six C-shaped bending magnets, 12 quadrupoles, two skew quadrupoles, four sextupoles, eight two-plane correctors, and two compensation solenoids.

For the first part of the injection Transfer Line (TL) of the antiprotons from the AD into ELENA, the consolidated transfer line 7000 will incorporate two $40^{\circ} \mathrm{H}$-shaped bending magnets, one existing CTF3 quadrupole, three ELENA ring-type quadrupoles, and two ELENA ring-type two-plane correctors.

In the ELENA main bending magnets, the magnetic flux densities will be $0.36 \mathrm{~T}$ at injection and $0.05 \mathrm{~T}$ at extraction. It is challenging to meet the required field quality of $2 \times 10-4$, because it means that, for example, at extraction the average field variation inside the requested good-field region must be below $\pm 0.1 \mathrm{G}$.

To accommodate this request, the transfer function of the bending magnet is optimized such that the influence of the non-linear BH curve is minimized, by maximizing the relative permeability of the magnets yoke. 
Two strategies are adopted to reach this goal: use high silicon content electrical steel, and dilute the magnetic material by placing some non-magnetic austenitic steel between the electrical steel laminations.

At the time of writing this document, the status of the magnets projects is as shown in table 1 .

Table 1. Status of the ELENA magnets

\begin{tabular}{|l|l|}
\hline \multicolumn{1}{|c|}{ Magnet } & \multicolumn{1}{|c|}{ Status } \\
\hline Ring Dipoles & $\begin{array}{l}\text { Contract is placed. Final manufacturing design report was submitted by } \\
\text { the contractor and is under approval. The contract performance is } \\
\text { according to schedule. }\end{array}$ \\
\hline $\begin{array}{l}\text { Tranfer Line } \\
\text { Dipoles }\end{array}$ & $\begin{array}{l}\text { Contract is placed. Final manufacturing design report was submitted by } \\
\text { the contractor and is under approval. The contract performance is } \\
\text { according to schedule. }\end{array}$ \\
\hline Quadrupoles & $\begin{array}{l}\text { The prototypes were built, measured and a decision on the yoke material } \\
\text { was taken. The invitation to tender will be sent out October 30 }\end{array}$ \\
\hline Sextupoles & Technical documentation is prepared, drawings are being updated. \\
\hline $\begin{array}{l}\text { Compensation } \\
\text { Solenoids }\end{array}$ & $\begin{array}{l}\text { Design and technical documentation are prepared, waiting for the final } \\
\text { approval of the ELENA project. }\end{array}$ \\
\hline $\begin{array}{l}\text { H/V corrector } \\
\text { magnets }\end{array}$ & $\begin{array}{l}\text { Prototype under construction, price enquiry is foreseen in December } \\
\text { 2014. }\end{array}$ \\
\hline Injection kicker & $\begin{array}{l}\text { Existing magnet to be re-used. New vacuum tank under manufacture at } \\
\text { CERN. Power generators to be relocated in adjacent building. }\end{array}$ \\
\hline Injection septum & $\begin{array}{l}\text { Existing magnet to be re-used. New power supply and control } \\
\text { electronics to be purchased. }\end{array}$ \\
\hline
\end{tabular}

\subsection{Electrostatic transfer lines components}

The very low kinetic energy of the beam to be extracted from ELENA makes it possible to consider using electrostatic optics elements. The advantages of electrostatic with respect to magnetic elements are good field shaping and stray field shielding possibilities, low power consumption, simple construction and low cost.

The electrostatic elements to be used are of four different kinds: the fast switches (used for ejection from the ELENA ring and for switching transfer lines), the static bends in the transfer lines, the steering dipoles in the transfer lines, and the quadrupoles of the transfer lines. Another electrostatic element (called ion switch) is to be implemented at the crossing of the injected and ejected beam. This device will also be used to inject $\mathrm{H}^{+}$or $\mathrm{H}^{-}$ions produced by the commissioning source via both the injection and extraction channel into the ELENA ring.

The design is handled at CERN and production is also planned to be carried-out in the CERN mechanical workshop. At the time of writing this document, the status of the electrostatic transfer lines main components is as shown in table 2. 
Table 2. Status of the electrostatic elements

\begin{tabular}{|l|l|}
\hline \multicolumn{1}{|c|}{ Element } & \multicolumn{1}{|c|}{ Status } \\
\hline Fast switches & $\begin{array}{l}\text { Design is complete, technical drawings are ready. Production planned to } \\
\text { start in January 2015 }\end{array}$ \\
\hline Bending elements & $\begin{array}{l}\text { Envelope is defined, electrostatic elements shapes are known. Technical } \\
\text { design to be ready for mid-2015. These elements are only needed in } \\
2017 .\end{array}$ \\
\hline $\begin{array}{l}\text { Quadrupoles and } \\
\text { steering dipoles }\end{array}$ & $\begin{array}{l}\text { Design is complete, technical drawings are ready. Production planned to } \\
\text { start in November 2014 }\end{array}$ \\
\hline Ion switch & $\begin{array}{l}\text { Production is ongoing at CERN. Testing is planned in May 2015 with } \\
\text { ELENA ion source }\end{array}$ \\
\hline
\end{tabular}

\subsection{Beam instrumentation}

Many beam diagnostics systems will be needed to measure the antiproton beam parameters from ejection from the AD up to the experiments situated in the different experimental zones. The intensity, position, profiles, tune, and cooling performance need to be monitored in order to provide the experiments with antiproton beams with the desired characteristic. A very short description of these systems is given below and the status is reviewed.

The BTV (Beam TV) system is being developed that will be capable of measuring the beam position and size just before the injection kicker and at the first turn in the ring. The proposed set-up consists of two distinct systems each incorporating a $6 \mathrm{~cm} \times 4 \mathrm{~cm}$ screen, a CCD camera, a filter wheel, optical elements, and a pneumatic in/out movement. The development of the BTV system is complete and production is expected to start early 2015 .

The ELENA orbit measurement system will be based on 20 Beam Position Monitors (BPMs) made of stainless steel, with vacuum as dielectric, and due to space constraints, mounted inside quadrupoles and dipoles. A prototype was built, and tests are being analysed. The production of the BPM's is expected to start in January 2015.

The profile of the circulating beam will be measured destructively, using a scraper system. In this device, a set of 4 blades is moved quickly across the beam and creates a particle shower due to the interaction of the beam with the blade. Simultaneous detection of the intensity of the particle shower outside the vacuum chamber with a scintillator/photomultiplier assembly gives an image of the beam profile. The design of the scraper is still in progress.

The ELENA Base-Band tune measurement system will be very similar to that of the AD, LEIR, PSB, and PS, based on the direct diode detection principle [1]. The technical design is in progress, and the manufacture is expected during first half of 2015.

Concerning the Longitudinal Pick-up and Shottky diagnostic system, a rescaled and adapted version of the ultra-low-noise AC beam current transformer deployed in the AD [2-4] will be used. In total, four such transformers will be installed in the ELENA ring and the transfer lines. Two transformers (one high-frequency and one low-frequency version) will be included in the ring and one low- 
frequency device will be installed in each ejection line. The design of the system is nearing completion, some of the material was already ordered.

The repetition rate of antiprotons extracted from ELENA is relatively low $(0.01 \mathrm{~Hz})$, and so to facilitate rapid tuning of the transfer lines, the location of the beam at several positions along the beam lines must be measured simultaneously. A set of micro-wire Beam Profile Monitors will be installed for this purpose. They are based on the devices that have been used by the ASACUSA collaboration since 1999 to measure $100 \mathrm{keV}$ antiproton or proton beams emerging from the Radio-Frequency Quadrupole Decelerator (RFQD). The design is now nearing completion and production is expected to start in January 2015.

\subsection{Radio-Frequency system}

The ELENA RF system that will assure the deceleration of the antiprotons has to provide RF voltage over a wide frequency range extending from $144 \mathrm{kHz}$ to above $2 \mathrm{MHz}$. At the lower frequency, the maximum required voltage is about $100 \mathrm{~V}$ (peak), while above $500 \mathrm{kHz}$ this value increases to $500 \mathrm{~V}$. To cover these requirements, a wideband cavity similar to those installed in the CERN PS Booster is perfectly adapted. It will consist of two magnetic alloy core, placed on either side of the accelerating gap and enclosed in a metallic box. The design of the ELENA RF system is nearing completion. Production is expected to start soon.

\subsection{Ion source}

For commissioning purposes, it is foreseen that an external source delivering $\mathrm{H}$ - ions and protons at $100 \mathrm{keV}$ will be installed near the ELENA ring. Protons can be injected into the machine via the extraction transfer line in the "reverse" sense but nominal polarity, in order to commission, for example, the magnetic system and measure the beam optics. $\mathrm{H}^{-}$ions will be injected via the normal injection transfer line into the ring with nominal sense and polarity. This will allow testing systems such as the electron cooler, where the nominal sense of the machine is of importance.

Due to the expected limited lifetime of $\mathrm{H}^{-}$ions in the ELENA machine, protons shall also be injected via the normal injection transfer line with nominal sense but reversed polarity of the machine, to assure efficient commissioning of the electron cooler.

An existing $50 \mathrm{kV} \mathrm{H} \mathrm{H}^{+}$source at the Research Centre Jülich, Germany, is being converted to fit ELENA needs and will be delivered to CERN in spring 2015.

\subsection{Infrastructure}

In order to make room for the ELENA machine, a number of elements have to be cleared from the AD hall. In this process, a new modular building was erected close to the $\mathrm{AD}$, to house the existing and future experiments control rooms. The 2 first stages of this building are now complete, and the Aegis, ATRAP and BASE experiments have already installed their control rooms there. The third stage will be completed for June 2015 so that ALPHA and ASACUSA control rooms can be relocated. This third stage will also include a control room for GBAR and a spare control room for a possible future experiment. The ELENA machine itself will be controlled from the existing AD control room.

In parallel, a new technical building was erected connected with the AD building to create space for element currently in the AD hall. This building was completed in June 2014. The existing AD kicker generators, the experiments cleaning rooms and the mechanical workshop will be transferred to this new technical building. 


\section{Project management and main milestones}

The project management aspects are led by the project leader and the technical coordinator. A work breakdown structure and a Project Breakdown Structure have been established at the early stage of the project.

The Quality Assurance Plan was drawn in 2012 and is being applied throughout the project, in particular for what concerns the internal and external reviews, the drawings control and approval processes etc.

The Technical Design Report [5] was issued in May 2013 and reviewed in October 2013. A cost and schedule review is planned to take place in November 2014.

The Earned Value Management system was adopted to monitor the advancement of the project.

The project has entered the manufacturing stage for the majority of the main components. The next major milestones include:

$-15^{\text {th }}$ December 2014: Start clearing the AD hall (Kicker generators, mechanical workshop, electrical switchboards, various barracks, some racks)

-January 2015: Start racks re-deployment

-March 2015: Shielding installation

-April 2015: Start magnets installation; ion source tests

-June 2016 - End 2016: commissioning of ELENA ring with ion source

-January 2017 - May 2017: installation of electrostatic transfer lines to existing experiments

-June 2017: Commissioning of transfer lines and first ELENA antiproton beams to experiments.

\section{References}

1. M. Gasior, "Faraday Cup Award: High sensitivity tune measurement using direct diode detection", CERN-ATS-2012-246 (2012)

2. G. Gonzalez, F. Pedersen, "An ultra-low noise AC beam transformer for deceleration and diagnostics of low intensity beams", PAC'99, New York (March 1999), THAR6, p.474 (2004)

3. M.E. Angoletta et al., "The new digital-receiver-based system for antiproton beam diagnostics", PAC'02, Chicago (June 2001), WPAH122, p. 2371 (2001)

4. M.E. Angoletta et al., "Upgrades to the digital receiver-based low-intensity beam diagnostics for CERN AD”, PAC'03, Knoxville (May 2003), WPPB023, p. 2461 (2003)

5. V. Chohan et al., "Extra Low ENergy Antiproton (ELENA) ring and its Transfer Lines: Design Report", 10.5170/CERN-2014-002 